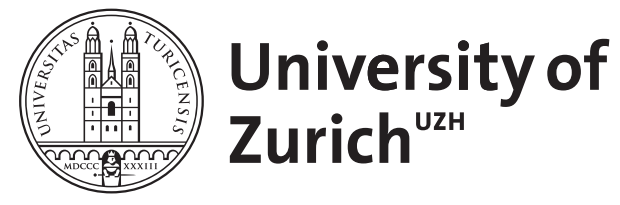

Zurich Open Repository and Archive

University of Zurich

University Library

Strickhofstrasse 39

CH-8057 Zurich

www.zora.uzh.ch

Year: 1996

\title{
Gaussian Estimates and Holomorphy of Semigroups on Lp Spaces
}

Hieber, Matthias

DOI: https://doi.org/10.1112/jlms/54.1.148

Posted at the Zurich Open Repository and Archive, University of Zurich

ZORA URL: https://doi.org/10.5167/uzh-154871

Journal Article

Published Version

Originally published at:

Hieber, Matthias (1996). Gaussian Estimates and Holomorphy of Semigroups on Lp Spaces. Journal of the London Mathematical Society, 54(1):148-160.

DOI: https://doi.org/10.1112/jlms/54.1.148 


\title{
GAUSSIAN ESTIMATES AND HOLOMORPHY OF SEMIGROUPS ON $L^{p}$ SPACES
}

\author{
MATTHIAS HIEBER
}

\section{Introduction}

Let $\Omega \subset \mathbb{R}^{n}$ be an open set and let $T_{p}=\left(T_{p}(t)\right)_{\geqslant 0}$ be consistent semigroups on $L^{p}(\Omega)$ for $1 \leqslant p<\infty$, with generators $A_{p}$. Assume that $T_{p_{0}}$ is an analytic semigroup of angle $\varphi$ for some $p_{0} \in(1, \infty)$. It is then natural to ask under which conditions the semigroups $T_{p}$ are analytic too. For the time being, suppose that $T_{p_{1}}$ and $T_{p_{2}}$ are contraction semigroups for given $p_{1}, p_{2} \in[1, \infty)$ and assume that $T_{p_{0}}$ is an analytic semigroup of contractions for some $p_{0} \in\left(p_{1}, p_{2}\right)$. Then, by standard arguments, $T_{p}$ is analytic for all $p \in\left(p_{1}, p_{2}\right)$. Observe however that $T_{p_{1}}$ is not analytic, in general.

In this paper we give a condition on the semigroup $T_{p_{0}}$ which forces the 'endpoint' semigroup $T_{p_{1}}$ to be analytic. More specifically, we prove that $T_{p_{1}}$ is analytic provided that $T_{p_{0}}$ satisfies an upper Gaussian estimate of order $m$. The case $p_{1}=1$ is of course of special interest and several results on $L^{1}$ holomorphy of semigroups have been obtained recently (cf. $[3,15])$. Our result generalizes in particular the above mentioned theorem of Ouhabaz which says that $T_{1}$ is an analytic semigroup on $L^{1}(\Omega)$ of angle $\pi / 2$ whenever $A_{2}$ is self-adjoint and $T_{2}$ admits a Gaussian estimate of order 2 .

Our result applies in particular to semigroups generated by second order elliptic differential operators $A$ subject to rather general boundary conditions. In fact, by a famous result of Agmon, Douglis and Nirenberg [1], we know that the $L^{p}$ realization of such a boundary value problem generates an analytic semigroup on $L^{p}(\Omega)$ for $1<p<\infty$, provided the top-order coefficients of $A$ belong to $B U C(\Omega)$. Observe, however, that their method does not extend to the space $L^{1}(\Omega)$. Assuming slightly more regularity on the coefficients of $A$, namely Hölder continuity, our result implies that the solutions of this kind of problem are governed by analytic semigroups also on the space $L^{1}(\Omega)$. We mention that our approach is based on the consequent use of the theory of integral operators.

The validity of Gaussian estimates for consistent semigroups $T_{p}$ is also of great importance for the problem concerning the $p$-independence of the spectrum $\sigma\left(A_{p}\right)$ of $A_{p}$. Indeed, as proved by Arendt [5], $\sigma\left(A_{p}\right)$ is independent of $p \in[1, \infty)$ whenever $A_{2}$ is self-adjoint and $T_{2}$ satisfies an upper Gaussian estimate of order 2. Applying his method to our situation we see that a certain component of the resolvent set of $A_{p}$ is independent of $p$. The general case dealing with the $p$-independence of $\sigma\left(A_{p}\right)$ for arbitrary generators of semigroups admitting Gaussian bounds remains, however, an open question.

Gaussian estimates for semigroups are, generally speaking, rather difficult to obtain. We therefore give in our second main result a characterization of analytic

Received 25 August 1994.

1991 Mathematics Subject Classification 47D03.

J. London Math. Soc. (2) 54 (1996) 148-160 
semigroups admitting a Gaussian estimate in terms of pointwise upper bounds on the kernel of a certain power of the resolvent. Those estimates might be verified more easily in concrete examples.

\section{Main results and examples}

Let $\Omega \subset \mathbb{R}^{n}$ be an open set, $p_{0} \in[1, \infty)$ and let $T$ be a $C_{0}$-semigroup on $L^{p_{0}}(\Omega)$ with generator $A$. In the following we always identify $L^{p_{0}}(\Omega)$ with a subspace of $L^{p_{0}}\left(\mathbb{R}^{n}\right)$ by extending functions by zero. Let $n \in \mathbb{N}, m \in \mathbb{N} \backslash\{1\}$ and define a constant $c_{m n}>0$ such that

$$
\frac{1}{c_{m n}} \int_{\mathbb{R}^{n}} \exp \left(\frac{-|x|^{m /(m-1)}}{4}\right) d x=1 .
$$

Moreover, define the family $\left(G_{p_{0}}(t)\right)_{t \geqslant 0}$ of operators on $L^{p_{0}}\left(\mathbb{R}^{n}\right)$ by $G_{p_{0}}(t) f:=k_{t} * f$, where

$$
k_{t}(x):=\frac{1}{c_{m n}} \frac{1}{t^{n / m}} \exp \left(\frac{-|x|^{m /(m-1)}}{4 t^{1 /(m-1)}}\right) \quad\left(t>0, x \in \mathbb{R}^{n}\right) .
$$

Since $k_{t} \in L^{1}\left(\mathbb{R}^{n}\right)$ for all $t>0$ it follows from Young's inequality that $\left\|G_{p_{0}}(t) f\right\|_{p_{0}} \leqslant$ $\left\|k_{t}\right\|_{1}\|f\|_{p_{0}}$. Furthermore, generalizing a notation of Arendt [5], we introduce the following definition.

DEFINITION 2.1. We say that the semigroup $T$ satisfies an upper Gaussian estimate of order $m$ if there exist constants $a \geqslant 0, M, b>0$ such that

for all $f \in L^{p_{0}}(\Omega)$.

$$
|T(t) f| \leqslant M e^{a t} G_{p 0}(b t)|f| \quad(t \geqslant 0)
$$

Notice that $G_{p_{0}}$ coincides with the Gaussian semigroup on $L^{p_{0}}\left(\mathbb{R}^{n}\right)$ provided $m=2$.

Furthermore, we assume that $E$ and $F$ are Banach spaces and that there exists a topological vector space $G$ such that $E \hookrightarrow G$ and $F \hookrightarrow G$. Then two operators $S_{E} \in \mathscr{L}(E)$ and $S_{F} \in \mathscr{L}(F)$ are called consistent if $S_{E} x=S_{F} x$ for all $x \in E \cap F$. We call two semigroups $T_{E}$ and $T_{F}$ on $E$ and $F$ consistent if $T_{E}(t)$ and $T_{F}(t)$ are consistent for all $t \geqslant 0$. Assume now that $T$ is a $C_{0}$-semigroup on $L^{p_{0}}(\Omega)$ which satisfies an upper Gaussian estimate of order $m \neq 1$. Then it is not difficult to verify that there exist consistent semigroups $T_{p}$ on $L^{p}(\Omega)$ (with $1 \leqslant p<\infty$ ) such that $T=T_{p_{0}}$ and

$$
\left|T_{p}(t) f\right| \leqslant M e^{a t} G_{p}(b t)|f| \quad\left(f \in L^{p}(\Omega), t \geqslant 0\right)
$$

(cf. Lemma 3.1). In order to state our results we need some more notation. For $\theta \in[0, \pi)$ put

$$
S_{\theta}:=\{z \in \mathbb{C} \backslash\{0\} ;|\arg z| \leqslant \theta\} \cup\{0\} \quad \text { and } S_{\theta}^{0}:=\{z \in \mathbb{C} \backslash\{0\} ;|\arg z|<\theta\} .
$$

Moreover, we call an operator $S \in \mathscr{L}\left(L^{p}(\Omega), L^{q}(\Omega)\right)$ for $1 \leqslant p, q \leqslant \infty$ an integral operator, if there exists a measurable function $K: \Omega \times \Omega \rightarrow \mathbb{C}$ such that for all $f \in L^{p}(\Omega), K(x, \cdot) f(\cdot) \in L^{1}(\Omega)$ x-a.e. and

$$
(S f)(x)=\int_{\Omega} K(x, y) f(y) d y \quad \text { x-a.e. }
$$


In that case $S$ is represented by the kernel and we write $S \sim K$. If in addition $|K|$ also defines an integral operator in $\mathscr{L}\left(L^{p}(\Omega), L^{q}(\Omega)\right)$, then $S$ is called a regular integral operator. It follows by standard arguments (cf. Proposition 3.3 below) that $T_{p}(t)$ is an integral operator, say $T_{p}(t) \sim K(t, \cdot, \cdot)$. We denote by $A_{p}$ the generator of $T_{p}$. Considering $e^{-a t} T(t)$ instead of $T(t)$, we may always assume that (2.1) is satisfied with $a=0$.

In our first result we show that, roughly speaking, $\left(\lambda-A_{p}\right)^{-l}$ for $l \in \mathbb{N}$ is a regular integral operator for all $\lambda$ belonging to a certain sector of the complex plane, provided that $T$ is analytic. More precisely, the following holds.

THEOREM 2.2. Suppose that $T$ is a bounded analytic $C_{0}$-semigroup on $L^{p}(\Omega)$ of angle $\varphi$ satisfying $a$ Gaussian estimate of order $m$ with $a=0$. Let $l \in \mathbb{N}, \theta \in[0, \varphi+\pi / 2)$ and $\lambda \in S_{\theta}^{0}$. Then $\left(\lambda-A_{p}\right)^{-l}$ is a regular integral operator and its kernel $K_{R}^{l}(\lambda, \cdot, \cdot)$ is given by

$$
K_{R}^{l}(\lambda, x, y)=\int_{\Gamma} e^{-\lambda z} \frac{z^{l-1}}{(l-1) !} K(z, x, y) d z,
$$

where $\Gamma$ is the ray $\left\{z \in \mathbb{C} ; z=|z| e^{-1 \phi}\right\}$ and $\phi \in[0, \varphi)$ is chosen such that $\theta-\phi<\pi / 2$. Moreover, there exist constants $C, \gamma>0$ such that the following estimates hold true.

(a) If $l<n / m$, then

$$
\left|K_{R}^{l}(\lambda, x, y)\right| \leqslant C \frac{1}{|x-y|^{n-m l}} e^{-\beta|x-y|}
$$

for all $x, y \in \Omega$ with $x \neq y$, all $\lambda \in S_{\theta}^{0}, \beta:=\left(\gamma m_{\theta}|\lambda|\right)^{1 / m}$ and $m_{\theta}:=\cos (\theta-\phi)$.

(b) If $l>n / m$, then

$$
\left|K_{R}^{l}(\lambda, x, y)\right| \leqslant C e^{-\alpha|x-y|}|\lambda|^{n / m-l}
$$

for all $x, y \in \Omega$, all $\lambda \in S_{\theta}^{0}$ and all $\alpha \in\left(0,\left(\gamma m_{\theta}|\lambda|\right)^{1 / m}\right)$.

(c) If $l=n / m$, then

$$
\left|K_{R}^{l}(\lambda, x, y)\right| \leqslant C e^{-\gamma|\lambda|^{1 / m}|x-y|}\left\{1+\log ^{+}\left(\frac{1}{|\lambda|^{1 / m}|x-y|}\right)\right\}
$$

for all $x, y \in \Omega$ with $x \neq y$ and all $\lambda \in S_{\theta}^{0}$.

Theorem 2.2 has two important consequences. The first one deals in particular with the $L^{1}$-analyticity of the consistent semigroup $T_{1}$ on $L^{1}(\Omega)$.

THEOREM 2.3. Suppose that $T$ is a bounded analytic $C_{0}$-semigroup on $L^{p_{0}}(\Omega)$ of angle $\varphi$ satisfying a Gaussian estimate of order $m$. Then $T_{p}$ is an analytic $C_{0}$-semigroup of angle $\varphi$ on $L^{p}(\Omega)$ for all $p \in[1, \infty)$.

REMARK. Theorem 2.3 generalizes in particular a recent result of Ouhabaz [15] saying that $T_{p}$ is an analytic semigroup of angle $\pi / 2$ on $L^{p}(\Omega)$ for $p \in[1, \infty)$ whenever $A_{2}$ is self-adjoint and $T$ admits an upper Gaussian estimate of order 2.

The second consequence of Theorem 2.2 concerns the $p$-independence of $\sigma\left(A_{p}\right)$, where $\sigma\left(A_{p}\right)$ denotes the spectrum of $A_{p}$. Let $\theta \in[0, \pi)$. Denote by $\rho_{\theta}\left(A_{p}\right)$ the connected component of the resolvent set $\rho\left(A_{p}\right)$ of $A_{p}$ which contains the sector $S_{\theta}^{0}$. It was shown 
by Arendt [5, Theorem 4.2] that $\rho_{\pi / 2}\left(A_{p}\right)$ is independent of $p \in[1, \infty)$ provided that $T$ admits a Gaussian estimate of order 2. Applying his theorem to our situation we obtain the following corollary.

COROLlary 2.4. Suppose that $T$ is a bounded analytic $C_{0}$-semigroup on $L^{p_{0}}(\Omega)$ of angle $\varphi$ satisfying a Gaussian estimate of order 2 . Let $\theta \in[0, \varphi+\pi / 2)$. Then $\rho_{\theta}\left(A_{p}\right)$ is independent of $p \in[1, \infty)$.

The above corollary needs some comment. Indeed, suppose that $A_{2}$ is self-adjoint and that $T_{2}$ satisfies an upper Gaussian estimate of order 2. Then, trivially $\rho\left(A_{2}\right)$ is connected and hence by Arendt's theorem, $\sigma\left(A_{p}\right)$ is independent of $p \in[1, \infty)$. The general case dealing with the $p$-independence of $\sigma\left(A_{p}\right)$ for arbitrary generators of semigroups admitting an upper Gaussian estimate, however, remains open.

Considering powers of the resolvent rather than the resolvent itself in Theorem 2.2 we are able to characterize analytic semigroups admitting a Gaussian bound in terms of a pointwise upper bound on the kernel of a certain power of the resolvent.

THEOREM 2.5. Let $T$ be a bounded analytic $C_{0}$-semigroup on $L^{p_{0}}(\Omega)$ of angle $\varphi$ with generator $A$. Then the following assertions are equivalent:

(a) $T$ satisfies an upper Gaussian estimate of order $m$;

(b) there exist an even integer $l>n / m+1$ and constants $M, c>0$ such that $(\lambda-A)^{-l}$ is a regular integral operator whose kernel $K_{R}^{l}(\lambda, \cdot, \cdot)$ satisfies

$$
\left|K_{R}^{l}(\lambda, x, y)\right| \leqslant M|\lambda|^{n / m-l} e^{-c|\lambda|^{1 / m}|x-y|}
$$

for all $x, y \in \Omega$ and all $\lambda \in S_{\theta}^{0}$, where $\theta \in(\pi / 2, \varphi+\pi / 2)$.

In the following we give three types of examples to which our theorems apply.

\section{EXAMPLES 2.6.}

\section{(A) Schrödinger operators}

Let $A=\Delta-V$ on $L^{2}\left(\mathbb{R}^{n}\right)$, where $V: \mathbb{R}^{n} \rightarrow \mathbb{R}$ is measurable and such that $V_{+} \in L_{\text {loc }}^{1}\left(\mathbb{R}^{n}\right)$ and $V_{-}$belongs to Kato's class (cf. [17]). Then $A$, equipped with a suitable domain, is a self-adjoint operator which generates a $C_{0}$-semigroup $T$ on $L^{2}\left(\mathbb{R}^{n}\right)$. It follows from [17; Proposition B.6.7] that $T$ satisfies a Gaussian estimate of order 2 . Denoting the consistent semigroup on $L^{p}$ by $T_{p}$, we conclude by Theorem 2.3 that $T_{p}$ is an analytic $C_{0}$-semigroup on $L^{p}\left(\mathbb{R}^{n}\right)$ of angle $\pi / 2$ for all $p \in[1, \infty)$.

(B) Elliptic operators on $L^{p}\left(\mathbb{R}^{n}\right)$ with Hölder continuous coefficients

Let $A=\sum_{|\alpha| \leqslant m} a_{\alpha}(x) D^{\alpha}, \rho \in(0,1), a_{\alpha} \in B U C^{\rho}\left(\mathbb{R}^{n}, \mathbb{C}\right)$ for $|\alpha|=m$ and $a_{\alpha} \in L^{\infty}\left(\mathbb{R}^{n}, \mathbb{C}\right)$ for $|\alpha| \leqslant m$. Suppose that there exists a constant $\delta>0$ such that

$$
\sup _{|\xi|=1} \operatorname{Re} \sum_{|\alpha|=m} a_{\alpha}(x)(\mathrm{i} \xi)^{\alpha}<-\delta \text { for all } x \in \mathbb{R}^{n} .
$$

Given $p \in(1, \infty)$ we define the $L^{p}$-realization $\mathscr{A}_{p}$ of $A$ by

$$
D\left(\mathscr{A}_{p}\right):=W_{p}^{m}\left(\mathbb{R}^{n}\right), \quad \mathscr{A}_{p} f:=A f \text { for all } f \in D\left(\mathscr{A}_{p}\right) .
$$

Then, as is well known, it follows that $\mathscr{A}_{p}$ generates an analytic $C_{0}$-semigroup $T_{p}$ on $L^{p}\left(\mathbb{R}^{n}\right)$ (for $\left.1<p<\infty\right)$ of some angle $\varphi \in(0, \pi / 2]$ (cf. [2]). Furthermore, it was shown 
by Friedman [9, Theorem 9.4.2] that $T_{p}$ satisfies an upper Gaussian estimate of order $m$. Denote by $T_{1}$ the consistent semigroup on $L^{1}\left(\mathbb{R}^{n}\right)$. Theorem 2.3 now implies that $T_{1}$ is an analytic $C_{0}$-semigroup on $L^{1}\left(\mathbb{R}^{n}\right)$ of angle $\varphi$.

We remark that one can easily generalize the above statement to the case of elliptic systems on $L^{1}\left(\mathbb{R}^{n}\right)^{N}$. In fact, let $\rho \in(0,1)$ and $a_{\alpha} \in B U C^{\rho}\left(\mathbb{R}^{n}, \mathscr{L}\left(\mathbb{C}^{N}\right)\right)$ for all $\alpha$ with $|\alpha|=m$. Assume that $\sup _{|\xi|=1} \operatorname{Re} \sigma\left(a_{m}(x, \xi)\right)<-\delta<0$ for some $\delta>0$ and all $x \in \mathbb{P}^{n}$, where $a_{m}(\cdot, \cdot)$ denotes the symbol of the principal part of $A=\sum_{|\alpha| \leqslant m} a_{\alpha}(x) D^{\alpha}$. Define $\mathscr{A}_{p}$ in $L^{p}\left(\mathbb{R}^{n}\right)^{N}$ for $1<p<\infty$ analogously to (2.3). It then follows from [4, Corollary 9.5], the above cited result of Friedman and the proof of Theorem 2.3 that the consistent semigroup $T_{1}$ on $L^{1}\left(\mathbb{R}^{n}\right)^{N}$ is analytic.

(C) Elliptic boundary value problems on $L^{1}(\Omega)$

Let $\Omega$ be a bounded domain in $\mathbb{R}^{n}$ such that $\partial \Omega \in C^{2+\rho}$ for some $\rho \in(0,1)$. Consider a differential operator $A$ of the form

$$
A(x, \partial):=-\sum_{1 \leqslant i, j \leqslant N} a_{i j}(x) \partial_{i} \partial_{j}+\sum_{1 \leqslant i \leqslant N} a_{i}(x) \partial_{i}+a_{0}(x),
$$

where $a_{i j}, a_{i}, a_{0} \in B U C^{\rho}(\Omega)$ and

$$
\sum_{1 \leqslant i, j \leqslant N} a_{i j}(x) \xi_{i} \xi_{j} \geqslant c|\xi|^{2}
$$

for all $x \in \mathbb{R}^{N}, \xi=\left(\xi_{1}, \ldots, \xi_{N}\right) \in \mathbb{R}^{N}$ and some constant $c>0$. Let $B(x, \partial):=$ $b(x) \cdot \nabla+b_{0}(x)$ be boundary operators such that $b=\left(b_{1}, \ldots, b_{n}\right), b_{i}, b_{0} \in C^{\rho}(\Omega)$ and $b(x) \cdot v(x) \geqslant c_{0}>0$, where $v(x)$ is the unit outward normal vector to $\partial \Omega$ at the point $x \in \partial \Omega$. Given $p \in(1, \infty)$, the operator

$$
D\left(\mathscr{A}_{p}\right):=\left\{u \in W_{p}^{2}(\Omega) ; B u=0\right\}, \quad \mathscr{A}_{p} u:=A u
$$

is called the $L^{p}$-realization of the boundary value problem $(A, B)$. Set

$$
\varphi_{A}:=\max _{x \in \tilde{\Omega}, \xi \in S^{n-1}} \operatorname{arct} g \frac{\left|\operatorname{Im} a_{n}(x, \xi)\right|}{\operatorname{Re} a_{\pi}(x, \xi)},
$$

where $a_{\pi}$ denotes the symbol of the principal part of $A$. Let $\varphi \in\left(\varphi_{A}, \pi / 2\right)$. Then it is known (cf. [2]) that $-\mathscr{A}_{p}$ generates an analytic semigroup $T_{p}$ on $L^{p}(\Omega)$ for $1<p<\infty$ of angle $\pi / 2-\varphi$. Furthermore, it is shown in [11] and [18] that the semigroup $T_{p}$ generated by $-\mathscr{A}_{p}$ satisfies an upper Gaussian estimate of order 2 . Denote by $T_{1}$ the consistent semigroup on $L^{1}(\Omega)$. It then follows from Theorem 2.3 that $T_{1}$ is an analytic semigroup on $L^{1}(\Omega)$ of angle $\pi / 2-\varphi$.

\section{Estimates for semigroup kernels}

Throughout this section we assume that $T$ is a $C_{0}$-semigroup on $L^{p_{0}}(\Omega)$ for some $p_{0} \in[1, \infty)$ which admits an upper Gaussian estimate of order $m$ and $a=0$. Considering $e^{-a t} T(t)$ instead of $T(t)$, the latter may be assumed without loss of generality.

We first prove two auxillary results. The first one is a modification of an argument due to Arendt [5, Assertion 4.3]. 
LEMMA 3.1. Let $1 \leqslant p<\infty$. Then there exists a consistent $C_{0}$-semigroup $T_{p}$ on $L^{p}(\Omega)$ such that $T=T_{p_{0}}$ and $\left|T_{p}(t) f\right| \leqslant M e^{a t} G_{p}(b t)|f|$ for $f \in L^{p}(\Omega), t \geqslant 0$.

Proof. Since $k_{t} \in L^{1}\left(\mathbb{R}^{n}\right)$, the operators $G_{p_{0}}(t)$ admit bounded extensions to $L^{p}\left(\mathbb{R}^{n}\right)$. Hence there exist consistent operators $T_{p}(t) \in \mathscr{L}\left(L^{p}\right)$ such that $T_{p_{0}}(t)=T(t)$ for all $t \geqslant 0$. The semigroup property of $T_{p}$ follows by density. We claim that $T_{p}$ is strongly continuous on $L^{p}$ (for $1 \leqslant p<\infty$ ). Note first that it suffices to show that $T_{p}(t) f \rightarrow f$ in $L^{p}$ as $t \rightarrow 0$ for $f \in L^{p} \cap L^{p_{0}}$. Therefore let $f \in L^{p} \cap L^{p_{0}}$ and let $t_{n} \rightarrow 0$. Put $f_{n}:=T_{p}\left(t_{n}\right) f$ and $g_{n}:=M e^{a t_{n}} G_{p}\left(b t_{n}\right) f$. Since it suffices to show that every subsequence of $f_{n}$ has a subsequence which converges to $f$, we may assume that $f_{n} \rightarrow f$ a.e. Observe that the family $\left(G_{p}(t)\right)_{t \geqslant 0}$ is strongly continuous in 0 . In fact, setting $\varphi(x):=$ $\left(1 / c_{m n}\right) \exp \left\{-|x|^{m /(m-1)} / 4\right\}$ and $\varepsilon:=t^{1 / m}$ it follows that $\left(1 / \varepsilon^{n}\right) \varphi(x / \varepsilon) * f \rightarrow f$ in $L^{p}\left(\mathbb{R}^{n}\right)$ as $\varepsilon \rightarrow 0$. Hence, by taking a subsequence, we may assume that $\left\|g_{n}-g_{n-1}\right\|_{p} \leqslant 2^{-n}$. Set $h:=\sum_{n \geqslant 2}\left|g_{n}-g_{n-1}\right|+\left|g_{1}\right|$. Then $h \in L^{p}\left(\mathbb{R}^{n}\right)$ and $\left|f_{n}\right| \leqslant g_{n} \leqslant h$ for all $n \in \mathbb{N}$. The claim finally follows now from the dominated convergence theorem.

Lemma 3.2. Let $E$ be a Banach space, $\varphi \in(0, \pi / 2]$ and $\alpha \in(0, \varphi]$. Assume that $B$ generates an analytic $C_{0}$-semigroup $S$ on $E$ of angle $\varphi$ and suppose that $e^{\mathrm{i} \alpha} B$ is the generator of a $C_{0}$-semigroup $U$ on $E$. Then $S\left(s e^{i \alpha}+t\right)=S(t) U(s)$ for $t>0, s>0$.

Proof. Since the resolvents of $B$ and $e^{\mathrm{i} \alpha} B$ commute, it follows that $U(s) S(t)=$ $S(t) U(s)$ for all $s, t>0$. Let $a, b>0$. Then the family $(V(t))_{t \geqslant 0}$ of operators on $E$ given by $V(t):=U(b t) S(a t)$ defines a $C_{0}$-semigroup on $E$. Denote the generator of $V$ by $C$. If $x \in D(B)$, then

$$
\frac{d}{d t} V(t) x=b e^{\mathrm{i} \alpha} B U(b t) S(a t) x+U(b t) a B S(a t) x=\left(b e^{\mathrm{i} \alpha}+a\right) B V(t) x .
$$

Hence $d /\left.d t V(t) x\right|_{t=0}=\left(b e^{i \alpha}+a\right) B x$ for $x \in D(B)$ and therefore $\left(b e^{i \alpha}+a\right) B \subset C$. Consequently $\left(b e^{i \alpha}+a\right) B=C$ and $V(t)=S\left(\left(b e^{i \alpha}+a\right) t\right)$ for all $t \geqslant 0$. In particular, $V(1)=S\left(b e^{i \alpha}+a\right)=U(b) S(a)$ for all $a, b>0$.

In the following proposition we collect some well-known facts on integral operators which will be used later on (see $[16$, Chapter IV; 6; 12] for proofs and references). For $1 \leqslant p<\infty, 1 / p+1 / p^{\prime}=1$, we put

$$
L^{\infty}\left[L^{p^{\prime}}\right]:=\left\{K: \Omega \times \Omega \longrightarrow \mathbb{C} \text { measurable; ess } \sup _{y \in \Omega}\left(\int_{\Omega}|K(x, y)|^{p^{\prime}} d x\right)^{1 / p^{\prime}}<\infty\right\} .
$$

Proposition 3.3. (a) Let $1 \leqslant p, q \leqslant \infty$ and let $S \in \mathscr{L}\left(L^{p}, L^{q}\right)$ be an integral operator represented by $K$. Let $S_{0} \in \mathscr{L}\left(L^{p}, L^{q}\right)$ such that $\left|S_{0} f\right| \leqslant S|f|$ for $f \in L^{p}(\Omega)$. Then $S_{0}$ is a regular integral operator and $\left|K_{0}(x, y)\right| \leqslant K(x, y)$ x-a.e., where $K_{0} \sim S_{0}$.

(b) Let $1 \leqslant p<\infty$ and consider the mapping

$$
\left(S_{K} f\right)(x)=\int_{\Omega} K(x, y) f(y) d y \quad\left(f \in L^{p}(\Omega)\right) .
$$

Then the mapping $K \mapsto S_{K}$ establishes an isometric isomorphism of $L^{\infty}(\Omega \times \Omega)$ onto $\mathscr{L}\left(L^{1}(\Omega), L^{\infty}(\Omega)\right)$ and of $L^{\infty}\left[L^{p^{\prime}}\right]$ onto $\mathscr{L}\left(L^{p}(\Omega), L^{\infty}(\Omega)\right)$ for $1<p<\infty$. 
In the sequel we show how to obtain upper bounds on the semigroup kernel $K(z, x, y)$ for complex times $z$. We hereby extend the approach of Davies [8, Section 3.4] to our situation.

Lemma 3.4. Let $\alpha \in(0, \pi / 2]$ and $\alpha^{\prime} \in(0, \alpha)$. Suppose that $T$ may be extended to a bounded, analytic $C_{0}$-semigroup on $L^{p_{0}}(\Omega)$ of angle $\alpha$. Then there exists a constant $C>0$ such that the kernel $K(z, \cdot, \cdot)$ of $T(z)$ satisfies

$$
|K(z, x, y)| \leqslant C /(\operatorname{Re} z)^{n / m} \quad \text { for all } z \in S_{\alpha^{\prime}} \backslash\{0\} \text { and all } x, y \in \Omega \text {. }
$$

Proof. We denote by $-A$ the generator of the semigroup $T$. Let $\varphi \in\left(\alpha^{\prime}, \alpha\right)$. It follows from [13, Theorem IX.1.23] that $-e^{\mathrm{i} \varphi} A$ and $-e^{-\mathrm{i} \varphi} A$ generate bounded $C_{0^{-}}$ semigroups on $L^{p_{0}}(\Omega)$. Denote these semigroups by $U_{+}$and $U_{-}$, respectively. Without of loss of generality we may assume that $\operatorname{Im} z \geqslant 0$. For $z \in S_{\alpha^{\prime}}\{\{0\} \cap[\operatorname{Im} z \geqslant 0]$ set $z=$ $s e^{i q}+t$, where $s, t \in \mathbb{R}$. Then by Lemma 3.2

$$
T(z)=T\left(s e^{\mathrm{i} \varphi}+t\right)=T(t) U_{+}(s)=T(t / 2) U_{+}(s) T(t / 2)
$$

and therefore

$$
\|T(z)\|_{\infty, 1} \leqslant\|T(t / 2)\|_{\infty, p_{0}}\left\|U_{+}(s)\right\|_{p_{0}, p_{0}}\|T(t / 2)\|_{p_{0}, 1} .
$$

By Proposition 3.3(a) we have that

$$
|K(t, x, y)| \leqslant \frac{M}{c_{m n}} \frac{1}{(b t)^{n / m}} \exp \left\{\frac{-|x-y|^{m /(m-1)}}{4(b t)^{1 /(m-1)}}\right\} \text { for all } t>0 \text { and all } x, y \in \Omega .
$$

Hence it follows from Young's inequality that

$$
\|T(t / 2)\|_{p_{0}, 1} \leqslant C t^{(n / m)\left(1 / p_{0}-1\right)}
$$

for some constant $C=C\left(M, p_{0}, n, m\right)$ and all $t>0$. Similarly, we conclude by Proposition $3.3(\mathrm{~b})$ that $\|T(t / 2)\|_{\infty, p_{0}} \leqslant C t^{(n / m)\left(1 / p_{0}^{\prime}-1\right)}$ for some constant $C=$ $C\left(M, p_{0}, n, m\right)$, all $t>0$ and $1 / p_{0}+1 / p_{0}^{\prime}=1$. Hence there exist a constant $C>0$ such that

$$
\|T(z)\|_{\infty, 1} \leqslant C / t^{n / m} \text { for all } t>0 .
$$

Proposition 3.3(b) now implies that

$$
|K(z, x, y)| \leqslant C / t^{n / m}
$$

for all $z \in S_{\alpha^{\prime}} \backslash\{0\}$ and all $x, y \in \Omega$. Notice furthermore that $t=\operatorname{Re} z-(\operatorname{Im} z / \sin \varphi) \cos \varphi$. Since $\operatorname{Im} z \leqslant \operatorname{Re} z \tan \alpha^{\prime}$ for all $z \in S_{\alpha^{\prime}} \backslash\{0\}$ it follows that

$$
t \geqslant \operatorname{Re} z\left(1-\tan \alpha^{\prime} \frac{\cos \varphi}{\sin \varphi}\right)=\operatorname{Re} z\left(1-\frac{\tan \alpha^{\prime}}{\tan \varphi}\right) \geqslant c \operatorname{Re} z
$$

for some constant $c>0$ and all $z \in S_{\alpha^{\prime}} \backslash\{0\}$. Combining this inequality with (3.2) we obtain

$$
|K(z, x, y)| \leqslant C /(\operatorname{Re} z)^{n / m}
$$

for all $z \in S_{\alpha^{\prime}} \backslash\{0\}$ and all $x, y \in \Omega$.

In order to obtain sharp estimates on the semigroup kernel for complex times $z$, we modify an idea of Davies (cf. [8, Theorem 3.4.8]). 
Lemma 3.5. Let $\beta \in(0, \pi / 2), \beta^{\prime} \in(0, \beta)$, and let $x, y \in \Omega$. Let $f(\cdot, x, y): S_{\beta} \rightarrow \mathbb{C}$ be a function which is analytic in $S_{\beta}^{0}$ and satisfies

$$
|f(z, x, y)| \leqslant \frac{C_{1}}{(\operatorname{Re} z)^{n / m}} \quad\left(z \in S_{\beta} \backslash\{0\}, x, y \in \Omega\right),
$$

where $C_{1}>0$ is a constant. Suppose that there exists a constant $a>0$ such that

$$
|f(r, x, y)| \leqslant \frac{C_{1}}{r^{n / m}} \exp \left\{-\frac{a|x-y|^{m /(m-1)}}{r^{1 /(m-1)}}\right\} \quad(r>0, x, y \in \Omega)
$$

Then there exists a constant $C_{2}=C_{2}\left(C_{1}, n, m, \beta, \beta^{\prime}\right)$ such that

$$
|f(z, x, y)| \leqslant \frac{C_{2}}{(\operatorname{Re} z)^{n / m}} \exp \left\{-\frac{a \sin \left(\frac{\beta-\beta}{m-1}\right)}{\sin (\beta / m-1)} \frac{|x-y|^{m /(m-1)}}{|z|^{1 /(m-1)}}\right\} \quad\left(z \in S_{\beta} \backslash\{0\}, x, y \in \Omega\right) .
$$

Proof. Set $D:=\{z \in \mathbb{C} ; 0 \leqslant \arg z \leqslant \beta\}$. For $z \in D$ and $x, y \in \Omega$ define the function $g$ by

$$
g(z, x, y):=\frac{1}{z^{n / m}} f(1 / z, x, y) \exp \left\{a e^{1(\pi / 2-\beta /(m-1))} z^{1 /(m-1)}|x-y|^{m /(m-1)} \frac{1}{\sin (\beta /(m-1))}\right\} .
$$

Then $|g(r, x, y)| \leqslant C_{1}$ for all $r>0$ and all $x, y \in \Omega$. Moreover, for $\theta \in[0, \beta]$ we have

$$
\left|g\left(r e^{1 \theta}, x, y\right)\right| \leqslant \frac{C_{1}}{(\cos \theta)^{n / m}} \exp \left\{a \frac{\cos \left(\frac{\pi}{2}-\frac{\beta}{m-1}+\frac{\theta}{m-1}\right)}{\sin (\beta /(m-1))} r^{1 /(m-1)}|x-y|^{m /(m-1)}\right\}
$$

for $r>0, x, y \in \Omega$. In particular, $\left|g\left(r e^{i \beta}, x, y\right)\right| \leqslant C_{1} /(\cos \beta)^{n / m}$ for all $r>0$ and all $x, y \in \Omega$. Observe that for $x, y \in \Omega$ and all $\varepsilon>0$ there exists $C>0$ (depending on $\varepsilon, x, y$ ) such that $|g(z, x, y)| \leqslant C e^{e z||^{2}}$ for all $z=r e^{i \theta}$ with $\theta \in(0, \beta)$ and $r$ sufficiently large. The Phragmèn-Lindelöf theorem (cf. [7, Corollary VI.4.4]) implies now that

$$
|g(z, x, y)| \leqslant C_{1} \frac{1}{(\cos \beta)^{n / m}} \quad \text { for all } z \in D, x, y \in \Omega
$$

Since

$$
f(z, x, y)=\frac{1}{z^{n / m}} g(1 / z, x, y) \exp \left\{\frac{-a e^{1(\pi / 2-\beta /(m-1))}|x-y|^{m /(m-1)}}{z^{1 /(m-1)} \sin (\beta /(m-1))}\right\}
$$

it follows that for $\arg z \in[-\beta, 0]$ we obtain

$$
\begin{aligned}
|f(z, x, y)| & \leqslant C_{1} \frac{1}{r^{n / m}} \frac{1}{(\cos \beta)^{n / m}} \exp \left\{\frac{-a \cos \left(\frac{\pi}{2}-\frac{\beta}{m-1}-\frac{\theta}{m-1}\right)|x-y|^{m /(m-1)}}{r^{1 /(m-1)} \sin (\beta /(m-1))}\right\} \\
& \leqslant \frac{C_{1}}{(\operatorname{Re} z)^{n / m}}\left(\frac{\cos \theta}{\cos \beta}\right)^{n / m} \exp \left\{\frac{-a \sin \left(\frac{\beta+\theta}{m-1}\right)|x-y|^{m /(m-1)}}{r^{1 /(m-1)} \sin (\beta /(m-1))}\right\} .
\end{aligned}
$$


Replacing $z$ by $\bar{z}$ in the above argument we obtain

$$
|f(z, x, y)| \leqslant \frac{C_{1}}{(\operatorname{Re} z)^{n / m}}\left(\frac{\cos \theta}{\cos \beta}\right)^{n / m} \exp \left\{\frac{-a \sin \left(\frac{\beta-|\theta|}{m-1}\right)|x-y|^{m /(m-1)}}{r^{1 /(m-1)} \sin (\beta /(m-1))}\right\}
$$

provided that $|\theta| \leqslant \beta$. Let now $z \in S_{\beta^{\prime}} \backslash\{0\}$. Then there exists a constant $C_{2}=$ $C_{2}\left(C_{1}, n, m, \beta, \beta^{\prime}\right)$ such that

$$
|f(z, x, y)| \leqslant \frac{C_{2}}{(\operatorname{Re} z)^{n / m}} \exp \left\{-a \frac{\sin \left(\frac{\beta-\beta}{m-1}\right)}{\sin \left(\frac{\beta}{m-1}\right)} \frac{|x-y|^{m /(m-1)}}{|z|^{1 /(m-1)}}\right\}
$$

for all $z \in S_{\beta} \backslash\{0\}$ and all $x, y \in \Omega$.

Combining Lemmas 3.4 and 3.5 we immediately obtain an upper bound on the semigroup kernel for complex times $z$.

Proposition 3.6. Assume that the conditions of Lemma 3.4 are satisfied. Then there exist constants $M, c>0$ such that the kernel $K(z, \cdot, \cdot)$ of $T(z)$ satisfies

$$
|K(z, x, y)| \leqslant \frac{M}{(\operatorname{Re} z)^{n / m}} \exp \left\{-\frac{c|x-y|^{m /(m-1)}}{|z|^{1 /(m-1)}}\right\}
$$

for all $z \in S_{\alpha^{\prime}} \backslash\{0\}$ and all $x, y \in \Omega$.

\section{Proofs of the main results}

We start the proof of Theorem 2.2 with an auxiliary result.

LEMMA 4.1. Let $E$ be a Banach space and let $S$ be a bounded holomorphic $C_{0^{-}}$ semigroup on $E$ of angle $\varphi \in(0, \pi / 2]$ with generator $-B$. Let $\theta \in[0, \varphi+\pi / 2)$ and choose $\phi \in[0, \varphi)$ such that $\theta-\phi<\pi / 2$. Denote by $\Gamma$ the ray $\left\{z \in \mathbb{C} ; z=|z| e^{-i \phi}\right\}$. Then

$$
(\lambda+B)^{-1}=\int_{\Gamma} e^{-\lambda z} S(z) d z \quad \text { for all } \lambda \in S_{\theta} \backslash\{0\} \cap[\operatorname{Im} z \geqslant 0] .
$$

The analogous result holds for $\lambda \in S_{\theta} \backslash\{0\} \cap\{z \in \mathbb{C} ; \arg z \leqslant 0\}$.

Proof. Let $\mu \in S_{\theta} \cap[\operatorname{Im} z \geqslant 0]$ and put $R(\mu):=\int_{\Gamma} e^{-\mu z} S(z) d z$. Then $R(\mu)$ is a well defined element of $\mathscr{L}(E)$. Moreover, the mapping $S_{\theta}^{0} \rightarrow \mathbb{C}, \lambda \mapsto R(\lambda)$ is holomorphic. Next, estimating the line-integral $\int_{\gamma_{R}} e^{-\lambda z} S(z) d z$, where $\gamma_{R}=\left\{R e^{\mathrm{i} \delta} ;-\phi \leqslant \delta \leqslant 0\right\}$, and letting $R \rightarrow \infty$ we see that

$$
R(\lambda)=\int_{0}^{\infty} e^{-\lambda t} S(t) d t=(\lambda+A)^{-1}
$$

provided that $\lambda>0$. The identity theorem for holomorphic functions now implies the assertion. 
Proof of Theorem 2.2. Let $\lambda \in S_{\theta}^{0}$ and assume without loss of generality that $\arg \lambda \geqslant 0$. Consider first the case where $l<n / m$. Setting $m_{\theta}:=\cos (\theta-\phi)$ we obtain, by Proposition 3.6,

$$
\begin{aligned}
\mid \int_{\Gamma} e^{-\lambda z} & \frac{z^{l-1}}{(l-1) !} K(z, x, y) d z \mid \\
\leqslant & \frac{M}{(l-1) !(\cos \phi)^{n / m}} \int_{\Gamma} e^{-m_{\theta} z z l \mid}|z|^{-n / m-1+l} \exp \left(\frac{-c|x-y|^{m /(m-1)}}{|z|^{1 /(m-1)}}\right) d|z| \\
= & \frac{M}{(l-1) !(\cos \phi)^{n / m}} \int_{\Gamma} \exp \left(-\left(m_{\theta}|z \lambda|+\frac{c|x-y|^{m /(m-1)}}{2|z|^{1 /(m-1)}}\right)\right. \\
& \times \exp \left(\frac{-c|x-y|^{m /(m-1)}}{2|z|^{1 /(m-1)}}\right)|z|^{l-n / m-1} d|z| .
\end{aligned}
$$

Setting $\beta:=\left(m m_{\theta}(c / 2)^{m-1}|\lambda|\right)^{1 / m}$ and observing that $m_{\theta}|z \lambda|+c|x-y|^{m /(m-1)} / 2|z|^{1 /(m-1)}$ $\geqslant \beta|x-y|$ we conclude that

$$
\begin{aligned}
\left|\int_{\Gamma} e^{-\lambda z} \frac{z^{l-1}}{(l-1) !} K(z, x, y) d z\right| \leqslant & \frac{M}{(l-1) !(\cos \phi)^{n / m}} e^{-\beta|x-y|} \\
& \times \int_{\Gamma} \exp \left(\frac{-c|x-y|^{m /(m-1)}}{2|z|^{1 /(m-1)}}\right)|z|^{-n / m-1+l} d|z| \\
\leqslant & C e^{-\beta|x-y|} \int_{0}^{\infty} \exp \left(\frac{-c|x-y|^{m /(m-1)} r}{2}\right) r^{n(1-1 / m)+l(1-m)-1} d r \\
\leqslant & C e^{-\beta|x-y|} \frac{1}{|x-y|^{n-l m}} .
\end{aligned}
$$

In particular we conclude by Young's inequality that

$$
y \mapsto \int_{\Gamma} e^{-\lambda z} \frac{z^{l-1}}{(l-1) !} K(z, x, y) d z u(y) \in L^{1}(\Omega)
$$

provided that $u \in L^{p}(\Omega)$. Hence it follows from the assumption, Lemma 4.1, (4.3) and Fubini's theorem that for $u \in L^{p_{0}}(\Omega)$ we have

$$
\begin{aligned}
\left(\lambda+A_{p_{0}}\right)^{-l} u(x) & =\int_{\Gamma} e^{-\lambda z} \frac{z^{l-1}}{(l-1) !} \int_{\Omega} K(z, x, y) u(y) d y d z \\
& =\int_{\Omega} \int_{\Gamma} e^{-\lambda z} \frac{z^{l-1}}{(l-1) !} K(z, x, y) d z u(y) d y .
\end{aligned}
$$

Hence $\left(\lambda-A_{p}\right)^{-l}$ is a regular integral operator for all $p \in[1, \infty)$ and its kernel is given by $K_{R}^{l}(\lambda, x, y)=\int_{\Gamma} e^{-\lambda z}\left(z^{l-1} /(l-1) !\right) K(z, x, y) d z$. The estimate (a) follows from (4.2). 
In order to prove (b) we set $u:=|\lambda||z|$ and deduce from (4.1) that

$$
\begin{aligned}
&\left|\int_{\Gamma} e^{-\lambda z} \frac{z^{l-1}}{(l-1) !} K(z, x, y) d z\right| \\
& \leqslant \frac{M}{(l-1) !(\cos \phi)^{n / m}} \int_{0}^{\infty} e^{-m_{\theta} u}\left(\frac{u}{|\lambda|}\right)^{l-1-n / m} \exp \left(\frac{-c|x-y|^{m /(m-1)}|\lambda|^{1 /(m-1)}}{u^{1 /(m-1)}}\right) \frac{1}{|\lambda|} d u \\
&= \frac{M}{(l-1) !(\cos \phi)^{n / m}|\lambda|^{n / m-l}} \int_{0}^{\infty} u^{l-1-n / m} e^{-\delta u} \\
& \times \exp \left(-\left[\left(m_{\theta}-\delta\right) u+\frac{c|x-y|^{m /(m-1)}|\lambda|^{1 /(m-1)}}{u^{1 /(m-1)}}\right]\right) d u
\end{aligned}
$$

for any $\delta \in\left(0, m_{\theta}\right)$. Setting $\alpha:=\left(m\left(m_{\theta}-\delta\right) c^{m-1}|\lambda|\right)^{1 / m}$ we obtain

$$
\begin{aligned}
\left|\int_{\Gamma} e^{-\lambda z} \frac{z^{l-1}}{(l-1) !} K(z, x, y) d z\right| & \leqslant \frac{M}{(l-1) !(\cos \phi)^{n / m}}|\lambda|^{n / m-l} e^{-\alpha|x-y|} \int_{0}^{\infty} u^{l-n / m-1} e^{-\delta u} d u \\
& \leqslant C(l, n, m, \theta)|\lambda|^{n / m-l} e^{-\alpha|x-y|}
\end{aligned}
$$

for all $x, y \in \Omega$ and all $\lambda \in S_{\theta}^{0}$. The rest of the proof can now be copied from Part (a).

In order to prove (c) we set $|z|:=|x-y|^{m} s$ and deduce from (4.1) that

$$
\left|\int_{\Gamma} e^{-\lambda z} \frac{z^{l-1}}{(l-1) !} K(z, x, y) d z\right| \leqslant \frac{M}{(l-1) !(\cos \phi)^{n / m}} \int_{0}^{\infty} e^{-m_{\theta}|\lambda||x-y|^{m} s}(1 / s) \exp \left(\frac{-c}{s^{1 /(m-1)}}\right) d s .
$$

Consider first the case where $|\lambda|^{1 / m}|x-y|<1$. Then $v:=\left(|\lambda \| x-y|^{m}\right)^{-1}>1$ and the integral on the right-hand side of (4.4) is bounded by

$$
\begin{aligned}
\int_{0}^{v} \frac{1}{s} \exp \left(-\frac{c}{s^{1 /(m-1)}}\right) d s+\int_{v}^{\infty} \frac{1}{s} \exp \left(-\frac{s m_{\theta}}{v}\right) d s \leqslant & \int_{0}^{1} \frac{1}{s} \exp \left(-\frac{c}{s^{1 /(m-1)}}\right) d s+\int_{1}^{v} \frac{1}{s} d s \\
& +\int_{1}^{\infty} \frac{1}{s} \exp \left(-s m_{\theta}\right) d s \\
& \leqslant C+\log v \leqslant C+m \log \left(\frac{1}{|\lambda|^{1 / m}|x-y|}\right)
\end{aligned}
$$

for some constant $C>0$. Second, if $|\lambda|^{1 / m}|x-y| \geqslant 1$, we set $w:=\left(|\lambda|^{1 / m}|x-y|\right)^{1-m}$. Then the integral on the right-hand side of (4.4) is bounded by

$$
\begin{aligned}
& \int_{0}^{w} \frac{1}{s} \exp \left(\frac{-c}{s^{1 /(m-1)}}\right) d s+\int_{w}^{\infty} \frac{1}{s} \exp \left(-\left[s m_{\theta}|\lambda \| x-y|^{m}+\frac{c}{s^{1 /(m-1)}}\right]\right) d s \\
& \leqslant \exp \left(\frac{-c}{2 w^{1 /(m-1)}}\right) \int_{0}^{w} \frac{1}{s} \exp \left(\frac{-c}{2 s^{1 /(m-1)}}\right) d s \\
& \quad+\exp \left(-m_{\theta}|\lambda|^{1 / m}|x-y| / 2\right)\left[\int_{w}^{1} \frac{1}{s} \exp \left(\frac{-c}{s^{1 /(m-1)}}\right) d s+\int_{1}^{\infty} \frac{1}{s} \exp \left(\frac{-m_{\theta} s}{2}\right) d s\right] \\
& \leqslant C(m, \theta, c) e^{-\gamma|\lambda|^{1 / m}|x-y|}
\end{aligned}
$$


for some $\gamma=\gamma(\theta, c)>0$. Now, combining (4.5) with (4.6) we see that there exists a constant $C=C(m, \theta, c, \gamma, l)$ such that

$$
\left|\int_{\Gamma} e^{-\lambda z} \frac{z^{l-1}}{(l-1) !} K(z, x, y) d z\right| \leqslant e^{-\gamma|\lambda|^{1 / m}|x-y|}\left\{1+\log ^{+}\left(\frac{1}{|\lambda|^{1 / m}|x-y|}\right)\right\}
$$

for all $x, y \in \Omega$ with $x \neq y$ and all $\lambda \in S_{\theta}^{0}$.

Finally, observe that $\int_{\mathbb{R}^{m}} e^{-|\lambda|^{1 / m}|x|} d x=C /|\lambda|$ for all $\lambda \in S_{\theta}^{0}$ and that

$$
\begin{aligned}
\int_{\mathbb{R}^{m}} e^{-|\lambda|^{1 / m}|x|} \log ^{+}\left(\frac{1}{|\lambda|^{1 / m}|x|}\right) d x & \leqslant C \int_{0}^{|\lambda|^{-1 / m}} e^{-|\lambda|^{1 / m} r} \log ^{+}\left(\frac{1}{|\lambda|^{1 / m} r}\right) r^{m-1} d r \\
& \leqslant \frac{C}{|\lambda|} \int_{0}^{\infty} s e^{-m s} d s \leqslant \frac{C}{|\lambda|}
\end{aligned}
$$

for all $\lambda \in S_{\theta}^{\mathbf{0}}$. Hence one may apply Fubini's theorem as in Part (a) in order to conclude that $\left(\lambda+A_{p}\right)^{-l}$ is an integral operator and that its kernel satisfies the required estimates.

Proof of Theorem 2.3. Let $\theta \in[0, \varphi+\pi / 2)$ and $a$ be as in Definition 2.1. Then there exists a constant $M>0$ such that

$$
\left\|\left(\lambda+a+A_{p}\right)^{-1}\right\|_{\mathscr{L}\left(L^{p}\right)} \leqslant M /|\lambda|
$$

for all $\lambda \in S_{\theta}^{0}$ and all $p \in[1, \infty)$. In fact, assuming $n \neq m$, this follows easily from Young's inequality and the estimates given in Theorem 2.2 (a) and (b), respectively. If $n=m$, then the above norm estimate follows from Young's inequality and (4.7). The claim follows now from the classical generation theorem for analytic semigroups (cf. [10, Theorem 1.5.4] or [14, A-II, Theorem 1.12]).

The assertion of Corollary 2.4 follows from [5, Theorem 4.2].

Proof of Theorem 2.5. By Theorem 2.2 we only have to prove the assertion (b) $\Rightarrow$ (a). To this end note that by integration by parts, the theorem of residues and the uniqueness theorem of Laplace transforms we have

$$
t^{l-1} T(t)=\frac{(l-1) !}{2 \pi \mathrm{i}} \int_{\Gamma} e^{t \lambda}(\lambda-A)^{-l} d \lambda \quad(t>0) .
$$

Here $\Gamma$ is defined by $\Gamma:=\Gamma_{-} \cup \Gamma_{0} \cup \Gamma_{+}$, where $\Gamma_{ \pm}:=\left\{r e^{ \pm 1 \alpha} ; r \geqslant R\right\}$ and $\Gamma_{0}:=\left\{\operatorname{Re}^{1 \Theta}\right.$; $|\Theta| \leqslant \alpha\}$ for suitable $R>0$ and $\alpha \in(\pi / 2, \varphi+\pi / 2)$. Clearly, the integral in (4.8) does not depend on the particular choice of $R$ and $\alpha$. For $t>0$ set

$$
K(t, x, y):=\frac{(l-1) !}{2 \pi \mathrm{i} t^{l-1}} \int_{\Gamma} e^{t \lambda} K_{R}^{l}(\lambda, x, y) d \lambda \quad(x, y \in \Omega) .
$$

The assumption (2.2) implies that

$$
\begin{gathered}
\left|\frac{(l-1) !}{2 \pi \mathrm{i} t^{l-1}} \int_{\Gamma_{ \pm}} e^{t \lambda} K_{R}^{l}(\lambda, x, y) d \lambda\right| \leqslant K \int_{R t}^{\infty} e^{u \cos \alpha} u^{n / m-l} d u \frac{1}{t^{n / m}} e^{-c R^{1 / m}|x-y|}, \\
\left|\frac{(l-1) !}{2 \pi \mathrm{i} t^{l-1}} \int_{\Gamma_{0}} e^{t \lambda} K_{R}^{l}(\lambda, x, y) d \lambda\right| \leqslant K R^{n / m-l+1} \frac{1}{t^{l-1}} e^{t R-c R^{1 / m}|x-y|}
\end{gathered}
$$

for some constant $K>0$, all $t>0$ and $x, y \in \Omega$. Choosing

$$
R:=\max \left\{\frac{1}{2^{m}}\left(\frac{c|x-y|}{t}\right)^{m /(m-1)}, t^{-1}\right\}
$$


we verify that there is a constant $M>0$ such that

$$
|K(t, x, y)| \leqslant \frac{M}{t^{n / m}} \exp \left\{-\frac{(c|x-y|)^{m /(m-1)}}{4 t^{1 /(m-1)}}\right\}
$$

for all $t>0$ and all $x, y \in \Omega$. Finally, by (4.8) we have

Since

$$
T(t) f(x)=\frac{(l-1) !}{2 \pi \mathrm{i} t^{l-1}} \int_{\Gamma} e^{t \lambda} \int_{\Omega} K_{R}^{l}(\lambda, x, y) f(y) d y d \lambda .
$$

$$
\begin{aligned}
\int_{\Omega}\left|\int_{\Gamma} e^{t \lambda} K_{R}^{l}(\lambda, x, y) d \lambda\right||f(y)| d y & \leqslant \frac{M_{1}}{t^{n / m}} \int_{\Omega} \exp \left\{-\frac{(c|x-y|)^{m /(m-1)}}{4 t^{1 /(m-1)}}\right\}|f(y)| d y \\
& \leqslant M_{2} G_{p_{0}}\left(c^{m /(m-1)} t\right)|f|(x)
\end{aligned}
$$

for some $M_{1}, M_{2}>0$, it follows from Fubini's theorem and Proposition 3.3(a) that $T(t)$ is a regular integral operator whose kernel $K(t, \cdot, \cdot)$ satisfies (4.9). The proof is complete.

\section{References}

1. S. Agmon, A. Douglis and L. Nirenberg, 'Estimates near the boundary for solutions of elliptic partial differential equations satisfying general boundary conditions', Comm. Pure Appl. Math. 12 (1959) 623-727.

2. H. AmanN, Linear and quasilinear parabolic problems (Birkhäuser, Basel, 1995).

3. H. AMANN and J. EsCHER, 'Strongly continuous dual semigroups', preprint, 1994.

4. H. Amann, M. Hieber and G. SimonetT, 'Bounded $H_{\infty}$-calculus for elliptic operators', Differential Integral Equations 7 (1994) 613-653.

5. W. ARENDT, 'Gaussian estimates and interpolation of the spectrum in $L^{p}$ ', Differential Integral Equations 7 (1994) 1153-1168.

6. W. ARENDT and A. V. BukHVAlov, 'Integral representations of resolvents and semigroups', Forum Math. 6 (1994) 111-135.

7. J. B. Conway, Functions of one complex variable (Springer, New York-Heidelberg-Berlin, 1973).

8. E. B. Davies, Heat kernels and spectral theory, Cambridge Tracts in Mathematics 92 (University Press, Cambridge, 1989).

9. A. Friedman, Partial differential equations of parabolic type (Prentice Hall, New Jersey, 1964).

10. J. A. Goldstein, Semigroups of linear operators and applications (Oxford University Press, New York, 1985).

11. S. D. IVASISIEN, 'Green's matrices of boundary value problems for Petrovskii parabolic systems of general form, I', Math. USSR-Sb. 42 (1982) 93-144.

12. L. V. Kantorovich and G. P. Akilov, Functional analysis (Nauka, Moscow, 1977; Pergamon Press, Oxford, 1982).

13. T. Kato, Perturbation theory of linear operators (Springer, Berlin-Heidelberg-New York, 1966).

14. R. NAGEL (ed.), One-parameter semigroups of positive operators, Lecture Notes in Mathematics 1184 (Springer, Berlin, 1985).

15. E. OuhaBaz, 'Propriétés d'ordre et de contractivité des semi-groupes avec applications aux opérateurs elliptiques', Ph.D. Thesis, Besançon, 1992.

16. H. H. SCHAEFER, Banach lattices and positive operators (Springer, Berlin, 1974).

17. B. Simon, 'Schrödinger semigroups', Bull. Amer. Math. Soc. 7 (1982) 447-526.

18. V. A. Solonnikov, 'On boundary value problems for linear parabolic systems of differential equations of general form', Trudy Mat. Inst. Steklov 83 (1965) 3-163; translated into Proc. Steklov Inst. Math. 83 Boundary value problems of mathematical physics, III (American Mathematical Society, Providence, 1967).

Universität Zürich

Mathematisches Institut

Winterthurerstrasse 190

CH-8057 Zürich

Switzerland 